\title{
A CLASS OF FINITE GROUPS WITH ZERO DEFICIENCY
}

\author{
by J. W. WAMSLEY
}

(Received 8th December 1972)

\section{Introduction}

Let $G$ be a finite group generated by $n$ elements and defined by $m$ relations, then $G$ has a presentation, $G=\left\{x_{1}, \ldots, x_{n} \mid R_{1}, \ldots, R_{m}\right\}=F / R$, where $F$ is free on generators $x_{1}, \ldots, x_{n}$ and $R$ is the normal closure in $F$ of $R_{1}, \ldots, R_{m}$. The deficiency of this presentation is $n-m$. Since $G$ is finite the deficiency is non-positive and the deficiency of $G$ is the maximal over the deficiencies of all presentations for $G$.

The torsion part of $R /[F, R]$ is a presentation invariant and is known as the Schur multiplicator of $G$. It is an open question as to whether a finite $p$-group with trivial Schur multiplicator has zero deficiency. One way to obtain a finite $p$-group, $G_{p}$, with trivial multiplicator is to take a finite group, $G$, with zero deficiency and let $G_{p}$ be the maximal $p$-factor of $G$.

One of the few known classes of finite groups with zero deficiency is given by Macdonald (1). A member of this class is presented by

$$
G(\alpha, \beta)=\left\{a, b \mid c=a^{-1} b^{-1} a b, c^{-1} a c=a^{\alpha}, c b c^{-1}=b^{\beta}\right\} .
$$

In this paper we show that for $p$ an odd prime, the maximal $p$-factor of $G(\alpha, \beta)$ has zero deficiency.

\section{Preliminaries}

In this section we give some results concerning the deficiency of factor groups of groups with zero deficiency. The following theorem (2, Theorem 2.1) is stated without proof.

Theorem 2.1. Let $G$ be a finite p-group with presentation $G=F / R$ and suppose the vector space $R /[F, R] R^{p}$ has dimension $m$. If we take any set of $m$ elements $R_{1}, \ldots, R_{m}$, of $R$, linearly independent in $R$ modulo $[F, R] R^{p}$ and let $K=F / S$, where $S$ is the closure of $R_{1}, \ldots, R_{m}$ in $F$, then $G$ is the maximal $p$ factor group of $K$, in the sense that if $A$ is a finite p-group which is a factor group of $K$ then $A$ is a factor group of $G$.

Corollary 2.2. Let $M=\left\{x_{1}, \ldots, x_{n} \mid R_{i_{1}}, \ldots, R_{i_{t}}\right\}$ where $R_{i_{1}}, \ldots, R_{i_{t}}$ is a subset of $R_{1}, \ldots, R_{m}$. If $M$ is a finite p-group then $G=K$.

Proof. $K$ is a factor of $M$ and hence $K$ is a finite $p$-group. 
Lemma 2.3. Let $G=\left\{x_{1}, \ldots, x_{n} \mid R_{1}, \ldots, R_{m}\right\}=F / R$ and

$$
G / N=\left\{x_{1}, \ldots, x_{n} \mid R_{1}, \ldots, R_{m}, S_{1}, \ldots, S_{u}\right\}=F / S
$$

then if $R_{i_{1}}, \ldots, R_{i_{\mathrm{t}}}$ are linearly independent in $S$ modulo $[F, S] S^{p}$ they are linearly independent in $R$ modulo $[F, R] R^{p}$.

Proof. The natural mapping $R /[F, R] R^{p}$ into $S /[F, S] S^{p}$ is a homomorphism and hence a linear transformation of the respective vector spaces.

Theorem 2.4. Let $G$ be a finite p-group with zero deficiency and $N$ a normal subgroup of $G$ contained in the derived group of $G$, then $G / N$ has trivial multiplicator if and only if $N=1$.

Proof. Take presentations for $G$ and $G / N$ to be

and

$$
G=\left\{x_{1}, \ldots, x_{n} \mid R_{1}, \ldots, R_{n}\right\}=F / R
$$

$$
G / N=\left\{x_{1}, \ldots, x_{n} \mid R_{1}, \ldots, R_{n}, S_{1}, \ldots, S_{u}\right\}=F / S,
$$

where $S_{i} \in F^{\prime}$, then $R_{1}, \ldots, R_{n}$ are linearly independent in $S$ modulo $[F, S] S^{p}$ and hence if $G / N$ has trivial multiplicator then $G / N$ is the maximal $p$-factor of $G$. However, $G$ is a finite $p$-group and therefore $G / N=G$. Conversely, if $G / N$ does not have trivial multiplicator then $G / N \neq G$ and hence $N \neq 1$ and the theorem is proved.

Hence if we give a presentation with zero deficiency which defines a finite $p$-group, $G$, and we show that $G_{p}(\alpha, \beta)$ is a factor group of $G$ and that

then $G=G_{p}(\alpha, \beta)$.

$$
G / G^{\prime} \cong G_{p} / G_{o}^{\prime}
$$

\section{The Main Results}

Let $p$ be an odd prime and consider the maximal $p$-factor, $G_{p}$, of a Macdonald group, i.e.,

$G_{p}=\left\{a, b \mid c=a^{-1} b^{-1} a b, c^{-1} a c=a^{1+j p^{r}}, c b c^{-1}=b^{1+k p^{s}}, a^{p^{x}}=1, b^{p^{\beta}}=1,\right\}$ where $(j, \mathrm{p})=1,(k, p)=1$, and $r, s, \alpha, \beta$ are some positive integers. We have for suitable $u, v$ and $w$ that

$$
c^{-u} a c^{u}=a^{1+p^{r}}, c^{-u v} b c^{u v}=b^{1+p^{s}} \text { and } c^{u}=a^{-u w} b^{-1} a^{u} b,
$$

where $(u, p)=1,(v, p)=1$ and $v$ is a power of a primitive root, $\gamma$, in the range $0<\gamma<2 p$. Further since $a^{p^{\alpha}}=1$, we may replace $w$ with $y=w+\delta$ where $p^{\alpha}$ divides $\delta$ and $v$ divides $y$. Note that $\alpha>r$.

Lemma 3.1. $y=1+t p^{r}$, where $1+2 t \not \equiv 0$ modulo $p$.

Proof. We have that $\left(1+j p^{r}\right)^{u} \equiv 1+p^{r}$ modulo $p^{\alpha}$. Hence $1+u j p^{r} \equiv 1+p^{r}$ modulo $p^{r+1}$ giving that $u j \equiv 1$ modulo $p$. Also

$$
b^{-1} a^{u} b=(a c)^{u}=c^{u} a^{\left(1+j p^{r}\right)\left(\left(1+j p^{r}\right)^{u}-1\right) / j p^{r}}
$$


and

$$
a^{u w} c^{u}=c^{u} a^{\left(1+j p^{r}\right)^{u_{u w}}}
$$

Equating right-hand sides gives that

$$
\left(1+j p^{r}\right)^{u-1} u w \equiv\left(\left(1+j p^{r}\right)^{u}-1\right) / j p^{r} \text { modulo } p^{\alpha} .
$$

Since $y \equiv w$ modulo $p^{r+1}$ we have

$$
\left(1+(u-1) j p^{r}\right) u y \equiv u+u(u-1) j p^{r} / 2 \text { modulo } p^{r+1},
$$

whence $y \equiv 1$ modulo $p^{r}$. Let $y=1+t p^{r}$ then substitution, cancellation and division by $p^{r}$ yields $u t+u(u-1) j / 2 \equiv 0$ modulo $p$, whence $2 t \equiv j-u j \equiv j-1$ modulo $p$ and we have $1+2 t \equiv j \neq 0$ modulo $p$ which proves the lemma. Hence we may choose, with a change of generators, a presentation for $G_{p}$ of the form

$G_{p}=\left\{a, b \mid c=a^{-y} b^{-1} a b, c^{-1} a c=a^{1+p^{r}}, c^{-v} b c^{v}=b^{1+p^{s}}, a^{p^{\alpha}}=1, b^{p^{\beta}}=1\right\}$, where $y=1+t p^{r}, 1+2 t \neq 0$ modulo $p,(v, p)=1, v$ divides $y$ and the only primes dividing $v$ lie in the range 0 to $2 p$. Let

$$
H=\left\{a, b \mid c=a^{-y} b^{-1} a b, c^{-1} a c=a^{1+p^{r}}, c^{-v} b c^{v}=b^{1+p^{s}}\right\},
$$

then $G$ is a factor group of $H$ and by the previous section when we show that $H$ is a finite $p$-group we have shown $G=H$ and hence $G$ is a two generatortwo relation finite $p$-group.

The following calculations are all in $H$.

Lemma 3.2. $a, b$ and $c$ have finite orders.

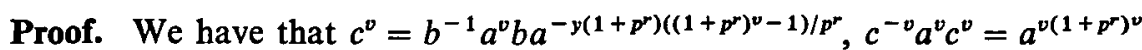
and $c^{-v} b c^{v}=b^{1+p^{s}}$ whence by Section 4 of (3) $a^{v}, b$ and $c^{v}$ generate a finite group since $v$ divides $y$.

Lemma 3.3. The order of $c$ divides the order of $a$.

Proof. Suppose the order of $a$ is $m$, then by raising the relation $b^{-1} a b=a^{y} c$ to the power $m$ we have $c^{m}=a^{-y\left(1+p^{r}\right)\left(\left(1+p^{r}\right)^{m-1}\right) / p^{r}}$, whence since the right-hand side commutes with $c$, the relation $c^{-1} a c=a^{1+p^{r}}$ gives that $m$ divides

$$
y\left(1+p^{r}\right)\left(\left(1+p^{r}\right)^{m}-1\right) \text {. }
$$

However, the power of $p$ which divides $m$ also divides $\left(\left(1+p^{r}\right)^{m}-1\right) / p^{r}$ and hence $c^{m}=1$.

Lemma 3.4. a has p-power order.

Proof. Since $c^{m}=1$, we have that $m$ divides $\left(\left(1+p^{r}\right)^{m}-1\right) / p^{r}$. Suppose $q$ is the smallest prime dividing $m$ and suppose $0<q<p$, then

$$
\left(1+p^{r}\right)^{m} \equiv 1 \text { modulo } q, \quad 1+p^{r} \not \equiv 1 \text { modulo } q,
$$

therefore some prime dividing $q-1$ divides $m$ or $q=2$. In either case $q=2$ 
since $q$ is the smallest prime dividing $m$. However, $m$ is odd and therefore $q=p$. Therefore $p$ is the smallest prime dividing $m$.

Similarly, if $q$ is a prime dividing $m$ and $0<q<2 p$ then $p$ divides $q-1$ or $p=q$ and hence $q=p$. Hence $(v, m)=1$.

Finally, suppose $q$ is the largest prime dividing $m$ and that $q \neq p$. Let $m^{\prime}=m / q$ then by Section 3 of (1) we have that $m$ divides $\left.\left(1+p^{r}\right)^{m^{\prime}}-1\right)$ and hence $m$ divides $\left.\left(1+p^{r}\right)^{m n^{\prime}}-1\right) / p^{r}$ giving that $b^{-1} a^{m^{\prime}} b=c^{m^{\prime}}$. Therefore

$$
b c^{m^{\prime}} b^{-1}=a^{m^{\prime}}=c^{m^{\prime}} b^{z}
$$

for some $z$. Conjugation by $c$ and elimination of $c^{m^{\prime}}$ gives that $a^{p^{r_{m^{\prime}}}}=b^{t^{\prime}}$ for some $t^{\prime}$ whence $a^{p^{r} m^{\prime}}$ is central and hence conjugation by $c$ gives that $a^{p^{2 r} m^{\prime}}=1$. However, this implies that $a^{m^{\prime}}=1$ and therefore $q=p$.

Lemma 3.5. We may solve the equation $c^{x}=a^{-x} b^{-1} a^{x} b$ where $(x, p)=1$.

Proof. $\quad b^{-1} a^{x} b=\left(a^{y} c\right)^{x}=c^{x} a^{y\left(1+p^{r}\right)\left(\left(1+p^{r}\right)^{x}-1\right) / p^{r}}$ and $a^{x} c^{x}=c^{x} a^{x\left(1+p^{r}\right) x}$

Therefore, if the order of $a$ is $p^{n}$, we need to solve the equation

$$
x\left(1+p^{r}\right)^{x-1} \equiv y\left(\left(1+p^{r}\right)^{x}-1\right) / p^{r} \text { modulo } p^{n} .
$$

This equation reduces to $f(x) p^{r} \equiv 0$ modulo $p^{n}$ where $f(x) \equiv 2 x(x-1-2 t)$ modulo $p$. Note that $y=1+t p^{r}$ where $1+2 t \neq 0$ modulo $p$. We show by induction on $n$ that $f(x) \equiv 0$ modulo $p^{n}$ has a solution coprime with $p$ for any $n$. For $n=1$ we have the solution $x=1+2 t$.

Assume that $f\left(x_{i}\right) \equiv 0$ modulo $p^{i}$, therefore $f\left(x_{i}\right)=k p^{i}$ for some $k$, where $f(x)=a_{0}+a_{1} x+a_{2} x^{2}+\ldots+a_{m} x^{m}$. We try $x=x_{i}+\delta p^{i}$ and we have

$$
\begin{aligned}
f(x)= & a_{0}+a_{1}\left(x_{i}+\delta p^{i}\right)+a_{2}\left(x_{i}+\delta p^{i}\right)^{2}+\ldots+a_{m}\left(x_{i}+\delta p^{i}\right)^{m} \\
\equiv & a_{0}+a_{1} x_{1}+a_{2} x_{i}^{2}+\ldots+a_{m} x_{i}^{m} \\
& \quad+a_{1} \delta p^{i}+2 a_{2} x_{i} \delta p^{i}+\ldots+m a \\
& \equiv k p^{i}+\delta p^{i} f^{\prime}\left(x_{i}\right) \text { modulo } p^{i+1} \\
\equiv & (k+\delta 2(1+2 t)) p^{i} \text { modulo } p^{i+1} .
\end{aligned}
$$$$
+a_{1} \delta p^{i}+2 a_{2} x_{i} \delta p^{i}+\ldots+m a_{m} x_{i}^{m-1} \delta p^{i} \text { modulo } p^{i+1}
$$

Hence we need to solve the equation

$$
k+2 \delta(1+2 t) \equiv 0 \text { modulo } p,
$$

whence $\delta=-k / 2(1+2 t)$ will do.

Lemma 3.6. $H$ is a finite nilpotent group.

Proof. $H$ is generated by $a^{x}$ and $b$ with relations, $c^{x}=a^{-x} b^{-1} a^{x} b$,

$$
c^{-x} a^{x} c^{x}=a^{x\left(1+p^{x}\right)^{x}}
$$

$c^{-x} b c^{x}=b^{z}$ for suitable $z$ and hence $H$ is a factor group of a Macdonald group and therefore nilpotent.

However $H / H^{\prime}$ is a finite $p$-group and therefore $H$ is a finite $p$-group. Also $H / H^{\prime} \cong G_{p} / G_{p}^{\prime}$ and hence $H=G_{p}$ giving 
Theorem 3.7. The maximal p-factor of a Macdonald group is a group with zero deficiency.

\section{REFERENCES}

(1) I. D. Macdonald, On a class of finitely presented groups, Canad. J. Math. 14 (1962), 602-613.

(2) J. W. WamsLeY, Minimal presentations for certain group extensions, Israel J. Math. 9 (1971), 459-463.

(3) J. W. WAMSLEY, A class of three-generator, three-relation, finite groups, Canad. J. Math. 22 (1970), 36-50.

The Flinders University of South Australia 